\title{
Proposta de indicador de sustentabilidade urbana-municipal relacionado ao consumo eletricidade que detecte ações de resposta à demanda ${ }^{1}$
}

\section{Proposal of an urban-municipal sustainability indicator related to electricity consumption that detects demand response actions}

\author{
Fonseca, André Luiz Amorim da'; Fernandes, Ricardo Augusto Souza²; \\ Teixeira, Bernardo Arantes do Nascimento ${ }^{3}$
}

\begin{abstract}
1 Instituto Federal de Educação, Ciência e Tecnologia de Mato Grosso (IFMT), doutorando pelo Programa de Pós Graduação em Engenharia Urbana pela Universidade Federal de São Carlos (UFSCar), Brasil, andre.fonseca@cba.ifmt.edu.br.

2 Universidade Federal de São Carlos (UFSCar), professor orientador pelo Programa de Pós Graduação em Engenharia Urbana, Departamento de Engenharia Elétrica (DEE), Brasil, ricardo.asf@gmail.com.

3 Universidade Federal de São Carlos (UFSCar), professor orientador pelo Programa de Pós Graduação em Engenharia Urbana, Departamento de Engenharia Civil (DCiv), Brasil, bernardo@ufscar.br.
\end{abstract}

\section{RESUMO}

Com o advento da tecnologia da informação e dos dispositivos de eletrônica embarcada, o cenário de construção ou adequação de cidades inteligentes se torna cada vez mais factível, possibilitando um gerenciamento dos recursos em tempo real. Neste contexto, surge a Resposta à Demanda (RD) que está inserida no Gerenciamento pelo Lado da Demanda (GLD) como maneira de reduzir picos e planificar a curva de consumo de eletricidade diário de uma cidade. Este trabalho efetua proposta de Indicador de Sustentabilidade Urbana (ISU) que possa mensurar impactos globais do uso de estratégias de GLD contornando limitações de alguns indicadores existentes estudados. A partir da análise dos indicadores selecionados foi possível perceber a ineficácia dos mesmos na observância de estratégias de RD que nas cidades inteligentes atuariam postergando investimentos em infraestrutura no sistema elétrico, amortecendo e desacelerando impactos ambientais provenientes da construção de novas usinas geradoras de energia elétrica, contribuindo para a sustentabilidade. Em sequência é proposto um ISU baseado em fatores que poderiam ser incluídos como parte de indicadores ou como indicadores individuais para que os impactos das estratégias fossem percebidos através desse ISU quando numericamente aplicado a uma cidade hipotética.

Palavras-chave: Gerenciamento pelo lado da demanda; Resposta à demanda; Indicadores de sustentabilidade urbana; Cidades inteligentes.

\section{ABSTRACT}

1 FONSECA, André Luiz Amorim; FERNANDES, Ricardo Augusto Souza; TEIXEIRA, Bernardo Arantes do Nascimento. Proposta de indicador de sustentabilidade urbana-municipal relacionado ao consumo eletricidade que detecte ações de resposta à demanda. In: II SIMPÓSIO NACIONAL DE GESTÃO E ENGENHARIA URBANA: SINGEURB, 2019, São Paulo. Anais... Porto Alegre: ANTAC, 2019. 
With the advent of information technology and embedded electronics devices, the construction or adaptation of smart cities becomes more and more feasible, allowing the management of resources in real time. In this context, the Demand Response(DR) within Demand Side Management (DSM) emerges as a way to reduce peaks and plan the daily consumption curve of a city. This paper makes a proposal of Urban Sustainability Indicator (USI) that can measure global impacts of the use of GLD strategies, circumventing limitations of some existing indicators studied. From the analysis of the selected indicators it was possible to perceive their inefficiency in the observance of DR strategies that in the smart cities would act by postponing investments in infrastructure in the electric system as well as cushioning and decelerating environmental impacts from the construction of new power plants, contributing to sustainability. Subsequently, an USI is proposed based on factors commonly applied in Electrical Engineering that could be included as part of indicators or as individual indicators so that the impacts of the strategies could be perceived through the proposed USI when numerically applied to a hypothetical city.

Keywords: Demand side management; demand response; urban sustainability indicators; smart cities.

\section{INTRODUÇÃO}

O uso da eletricidade constitui parte dos Indicadores de Sustentabilidade Urbana(ISU). Neste contexto, figura-se o Gerenciamento pelo Lado da Demanda(GLD), que considera o controle de consumo como variável, alterando o paradigma operativo pelo oferta de eletricidade.

Dentre técnicas de GLD destaca-se a Resposta à Demanda(RD) que consiste em controlar a demanda por eletricidade indireta (via tarifação) ou diretamente de forma a permitir que as concessionárias controlem remotamente cargas elétricas. Uma modalidade de RD vigente no Brasil é a tarifa branca, que usa de modulação tarifária com intuito de reduzir o consumo de eletricidade no período de ponta (ANEEL, 2016). Já o mercado RD por controle direto tem se desenvolvido tanto no Canadá quanto na Europa (Wong,2015; SEDC,2015; SEDC,2017).

A Figura 1 ilustra o estado dos programas de RD na Europa ao final de 2016.

Figura 1 - Mapa do desenvolvimento do mercado da DR na Europa

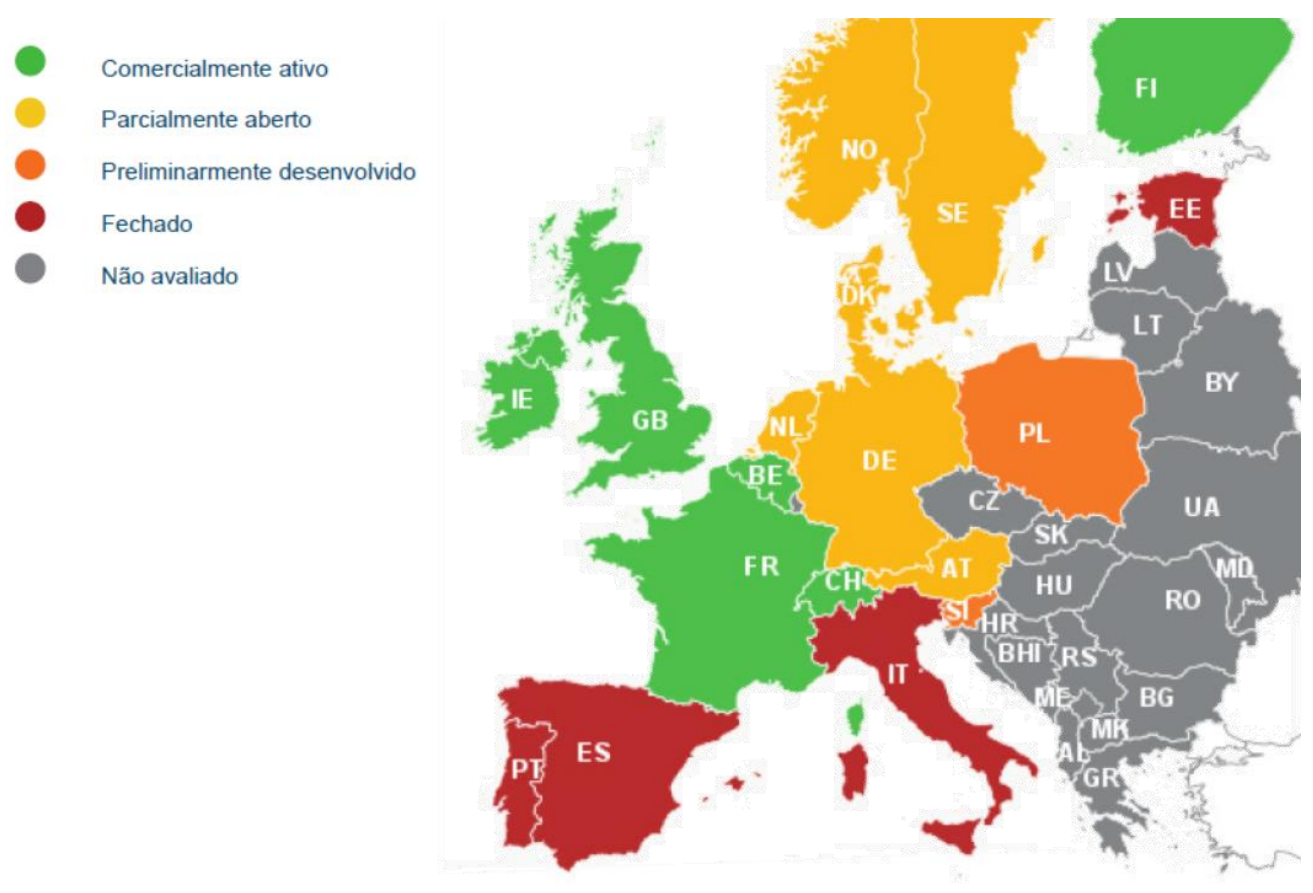

Fonte: Adaptado de SEDC(2017)

Esses tipos de programas tem como meta a planificação da curva de consumo diário, sendo um postergador da ampliação do sistema de suprimento. 
Segundo Bell e Morse (2008), qualquer indicador de sustentabilidade deve avaliar um sistema coletando dados que possam ser interpretados possibilitando uso conforme Figura 2.

Figura 2 - O conceito dos indicadores de sustentabilidade

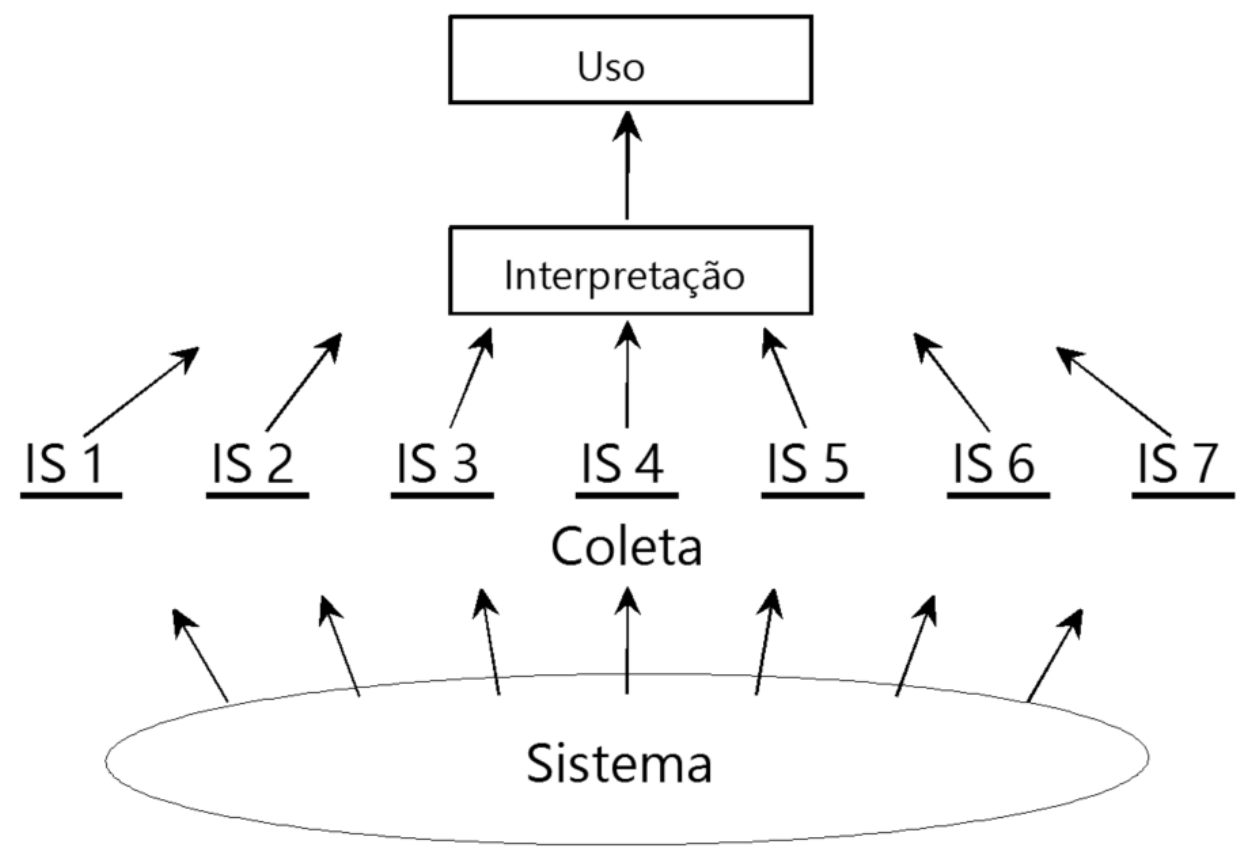

Fonte: Adaptado de Bell e Morse (2008)

Segundo Silva e Shimbo (2000) dentre as características fundamentais dos ISU figuram a conjunção técnica e política, consistência científica e a confiabilidade estatística. A NBR ISO 37120 (ABNT, 2017) apresenta vários ISU a nível internacional tratando de indicadores relacionados ao consumo de eletricidade com horizonte anual.

Assim, este trabalho possui como meta propor um novo ISU detecte a efetividade de programas de RD aplicados em uma cidade hipotética a partir da análise de alguns sistemas de ISU, a saber: o Sustainable Seattle (1998), de Seattle; o Indicators 21 , do Municipal Council of Environment and Sustainability (2012) de Barcelona e o Programa Cidades Sustentáveis, desenvolvido pela Rede Nossa São Paulo (2018).

\section{METODOLOGIA APLICADA}

Na presente proposta dos sistemas de ISU estudados foram separados os relacionados à eletricidade que demonstrem capacidade de detecção do uso de estratégias de GLD e RD. Em um segundo momento, fatores aplicados a sistemas elétricos são apresentados e mesclados com um ISU tradicional, através do que se deu a proposição de um novo ISU que detecte os impactos globais de GLD, apresentando também metodologias de coleta e cálculo.

\section{ISU ESTUDADOS}

Para cada sistema de ISU será apresentado quadro contendo principais ISU estudados ressaltando tema, tipo, nome, descrição, unidade de medida e componentes, divisão baseada no livro sobre indicadores de energia para o desenvolvimento sustentável (IAEA et al., 2005).

\subsection{Sustainable Seattle}

Os ISU da cidade de Seattle foram inspirados por uma conferência em 1990, com posterior 
organização de voluntários que criaram a Sustainable Seattle Network propondo ISU que mediriam a saúde urbana. A partir de reuniões com especialistas e voluntários, os indicadores foram democraticamente desenvolvidos e escolhidos para serem monitorados e auxiliarem nos processos decisórios. Tal iniciativa foi considerada pioneira (SUSTAINABLE SEATTLE, 1998). O Quadro 1 apresenta um resumo dos dois ISU analisados.

\section{Quadro 1- ISU selecionados do Sustainable Seattle}

\begin{tabular}{|c|c|c|c|c|c|}
\hline $\begin{array}{c}\text { Tema ou } \\
\text { Grupo }\end{array}$ & Tipo & $\begin{array}{c}\text { Nome do } \\
\text { indicador }\end{array}$ & Descrição do indicador & $\begin{array}{c}\text { Unidade de } \\
\text { medida }\end{array}$ & Componentes \\
\hline $\begin{array}{c}\text { População } \\
\text { e recursos }\end{array}$ & Social & $\begin{array}{c}\text { Uso de energia } \\
\text { renovável } \\
\text { não-renovável }\end{array}$ & $\begin{array}{c}\text { Série temporal do uso de } \\
\text { diferentes formas de } \\
\text { energia em cada ano }\end{array}$ & $\begin{array}{c}\text { Trilhões de BTU } \\
\text { no ano }\end{array}$ & $\begin{array}{c}\text { Gaturalina, eletricidade } \\
\text { renovável e } \\
\text { eletricidade não- } \\
\text { renovável }\end{array}$ \\
\hline Economia & Econômico & $\begin{array}{c}\text { Uso de energia } \\
\text { por dólar de } \\
\text { renda }\end{array}$ & $\begin{array}{c}\text { Série temporal de energia } \\
\text { por dinheiro norte } \\
\text { americano }\end{array}$ & $\begin{array}{c}\text { Milhares de BTU } \\
\text { por Dólar }\end{array}$ & $\begin{array}{c}\text { Milhares de BTU não- } \\
\text { renovável por Dólar } \\
\text { e milhares totais de } \\
\text { BTU por Dólar }\end{array}$ \\
\hline
\end{tabular}

Fonte: Sustainable Seattle (1998)

O uso de fontes renováveis pode ser impactado com estratégias de GLD que desviem o consumo para horário de maior disponibilidade de eletricidade renovável. Já a economia de energia por renda envolve aspectos do GLD por poder ser impactado pela eficiência energética. Entretanto, o melhor aproveitamento do sistema elétrico em distribuição diária não é detectado pelos ISU apresentados.

\subsection{Indicators 21}

Os ISU da cidade de Barcelona são mantidos pelo Conselho Municipal da Cidade de Barcelona, alinhado aos princípios de sustentabilidade propostos pela ONU (MUNICIPAL COUNCIL OF ENVIRONMENT AND SUSTAINABILITY, 2012). No Quadro 2, é apresentado o único ISU relacionado a eletricidade. Este indicador trata apenas do lado da oferta, sendo assim diametralmente oposta a GLD.

Quadro 2- ISU selecionados do Indicators 21

\begin{tabular}{|c|c|c|c|c|c|}
\hline $\begin{array}{c}\text { Tema ou } \\
\text { Grupo }\end{array}$ & Tipo & $\begin{array}{c}\text { Nome do } \\
\text { indicador }\end{array}$ & Descrição do indicador & $\begin{array}{c}\text { Unidade de } \\
\text { medida }\end{array}$ & Componentes \\
\hline $\begin{array}{c}\text { População } \\
\text { e Recursos }\end{array}$ & Social & $\begin{array}{c}\text { Uso de energias } \\
\text { renováveis } ~ \\
\text { produção de } \\
\text { energia solar }\end{array}$ & $\begin{array}{c}\text { Série temporal do uso de } \\
\text { energia renovável } \\
\text { percentual a cada ano; } \\
\text { energia equivalente } \\
\text { gerada nas usinas } \\
\text { fotovoltaicas }\end{array}$ & $\begin{array}{c}\text { População e } \\
\text { Recursos }\end{array}$ \\
\hline
\end{tabular}

Fonte: Municipal Council Of Environment And Sustainability (2012)

\subsection{Programa Cidades Sustentáveis}

O Programa Cidades Sustentáveis tem por objetivo auxiliar no desenvolvimento sustentável urbano-municipal. Tal programa estimula a aplicação dos ISU nas cidades a partir de comprometimento prévio de candidatos à prefeitura (REDE NOSSA SÃO PAULO, 2012). O Quadro 3 apresenta os indicadores estudados. 
Quadro 3- ISU selecionados do Programa Cidades Sustentáveis

\begin{tabular}{|c|c|c|c|c|c|}
\hline $\begin{array}{c}\text { Tema ou } \\
\text { Grupo }\end{array}$ & Tipo & $\begin{array}{l}\text { Nome do } \\
\text { indicador }\end{array}$ & Descrição do indicador & $\begin{array}{l}\text { Unidade de } \\
\text { medida }\end{array}$ & Componentes \\
\hline $\begin{array}{c}\text { Bens naturais } \\
\text { comuns }\end{array}$ & Ambiental & $\begin{array}{l}\text { Consumo de } \\
\text { energia } \\
\text { produzida por } \\
\text { fontes } \\
\text { renováveis }\end{array}$ & $\begin{array}{c}\text { Consumo de energia } \\
\text { produzida por fontes } \\
\text { renováveis sobre o total de } \\
\text { energia consumida }\end{array}$ & $\begin{array}{c}\text { Não } \\
\text { especificada }\end{array}$ & $\begin{array}{l}\text {-Hidrelétrica, } \\
\text { biomassa, solar, das } \\
\text { marés, das ondas e } \\
\text { eólica }\end{array}$ \\
\hline $\begin{array}{l}\text { Economia } \\
\text { Local } \\
\text { Dinâmica, } \\
\text { Criativa e } \\
\text { Sustentável }\end{array}$ & Econômico & $\begin{array}{c}\text { Eficiência } \\
\text { energética da } \\
\text { economia }\end{array}$ & $\begin{array}{l}\text { Razão entre Consumo } \\
\text { Interno Bruto de Energia e o } \\
\text { PIB, calculada para um ano } \\
\text { civil, a preços constantes, } \\
\text { com base no ano anterior }\end{array}$ & $\begin{array}{c}\text { Não } \\
\text { especificada }\end{array}$ & $\begin{array}{c}\text {-Carvão, } \\
\text { eletricidade, } \\
\text { petróleo, gás natural } \\
\text { e fontes de energia } \\
\text { renovável } \\
\text { disponíveis para } \\
\text { consumo }\end{array}$ \\
\hline $\begin{array}{l}\text { Consumo } \\
\text { Responsável } \\
\text { e Opções } \\
\text { de Estilo de } \\
\text { Vida }\end{array}$ & $\begin{array}{l}\text { População } \\
\text { e recursos }\end{array}$ & $\begin{array}{c}\text { Consumo total } \\
\text { de eletricidade } \\
\text { per capita }\end{array}$ & $\begin{array}{c}\text { Quantidade de } \\
\text { eletricidade consumida } \\
\text { pelos cidadãos (residencial, } \\
\text { industrial e outros) em um } \\
\text { ano em } \\
\text { todos os edifícios do } \\
\text { município, } \\
\text { independentemente da } \\
\text { razão para o uso }\end{array}$ & $\begin{array}{c}\text { Não } \\
\text { especificada }\end{array}$ & Não especificadas \\
\hline
\end{tabular}

Fonte: Rede Nossa São Paulo (2012)

Todos ISU do quadro tratam do consumo, podem detectar GLD, porém a distribuição do consumo ao longo do dia também não seria detectada.

\section{DETECÇÃO DA RD NO ISU PROPOSTO}

No âmbito da distribuição de eletricidade, alguns fatores são aplicados com o intuito de detectar a racionalidade de seu uso. Assim, a curva diária de demanda de uma cidade hipotética pode ser visualizada na Figura 3.

Figura 3 - Curva de carga de cidade hipotética

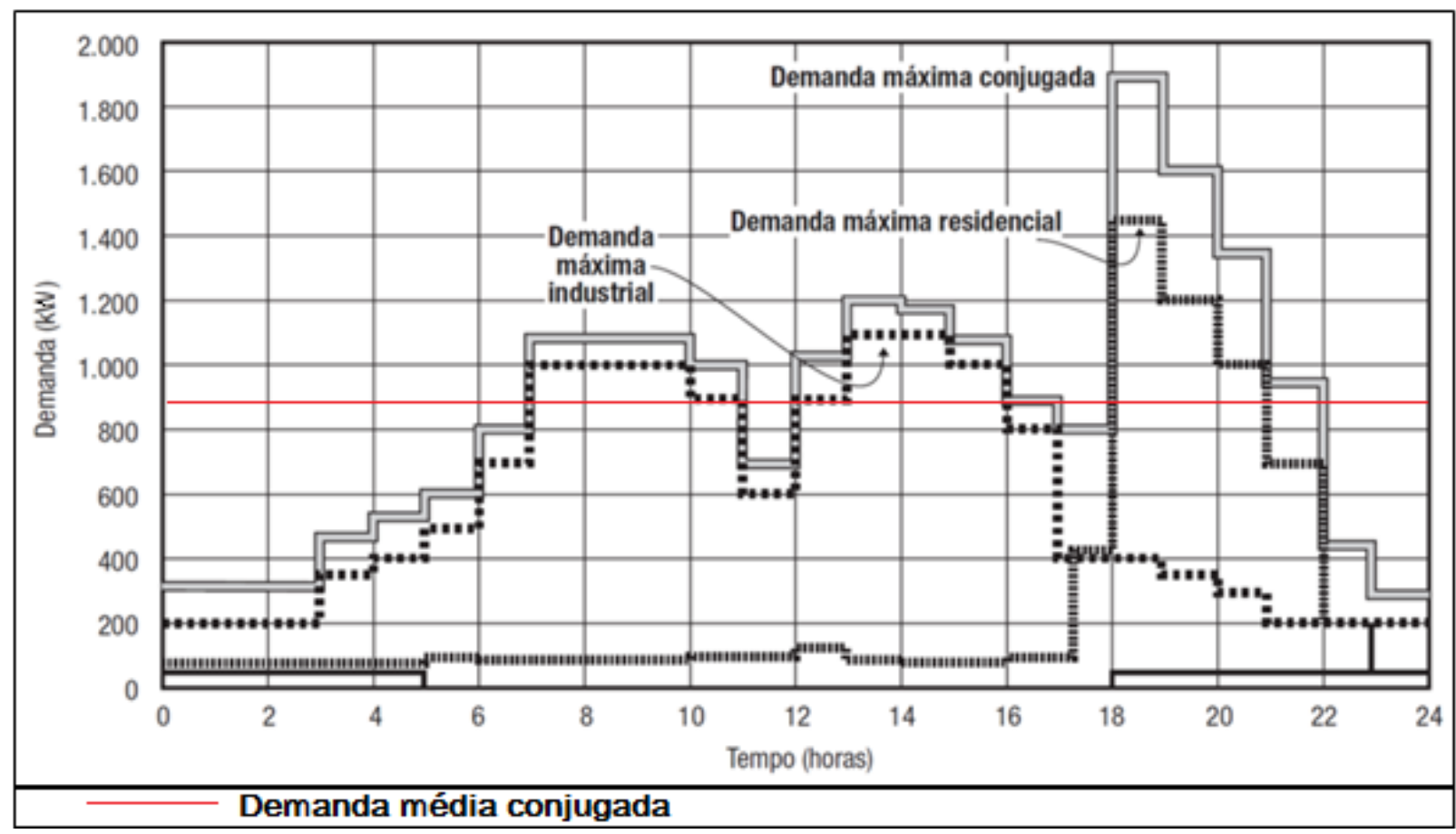

Fonte: Adaptado de Kagan, Oliveira e Robba(2005) 
A partir da análise da ilustração, notam-se diferenças horárias nos picos de demanda, sendo o consumo diário total conjugado de 21,045[MWh], com demanda média conjugada de $876,875[\mathrm{~kW}$ ] e máxima de 1,9[MW], entretanto se não ocorressem picos de consumo na curva haveriam ganhos a critério de sustentabilidade, mas os ISU apresentados não os detectariam. Para a proposição do ISU que melhor detecte-os será considerado o fator de carga, matematicamente apresentado e aplicado aos resultados expostos considerando 24 horas transcorridas, pela Equação(1):

$$
f_{\text {carga }}=\frac{D_{\text {média }}}{D_{\text {máxima }}}=\frac{\frac{\int_{0}^{\tau} d(t) \times d t}{\tau}}{D_{\text {máxima }}}=\frac{\int_{0}^{\tau} d(t) \times d t}{D_{\text {máxima }} \times \tau}=\frac{21,045 \times 10^{6}}{1,9 \times 10^{6} \times 24}=0,461513 \text { [adimensional] }
$$

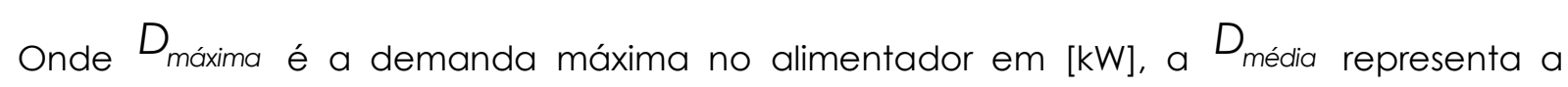
demanda média em $[\mathrm{kW}], d(t)$ é a demanda instantânea ou a demanda média discretizada em um tempo definido em [kW] e $\tau$ é o tempo total do cliclo de demanda analisada, na unidade de tempo considerada.

Para efetuar o contraste com o ISU tradicional foi utilizado o consumo de energia elétrica per capita, presente no terceiro indicador do Quadro 3 como uma relação anual e encontra-se implicitamente presente no segundo indicador do Quadro 1. A seguir, na Equação (2) é apresentado tal indicador para um dia, considerando uma cidade com 100.000 habitantes:

$$
C_{\text {diário-per-capita }}=\frac{\int_{0}^{\tau} d(t) \times d t}{N_{\text {habitantes-municíio }}^{\circ}}=\frac{21,045 \times 10^{6}}{100.000}=210,45\left[\frac{\mathrm{Wh}}{\text { hab } \times \text { dia }}\right]
$$

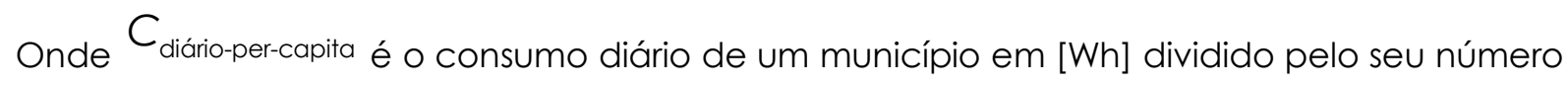
de habitantes. Considerando a cidade hipotética de 100.000 habitantes o $C_{\text {diário-per-capita seria }}$ de 210,45[Wh/(habitante.dia)].

A partir das equações apresentadas foi proposto novo indicador juntamente com metodologia de aplicação, factível pelo processo de digitalização das subestações elétricas e a crescente tendência para a consolidação das smart grids.

\section{ISU URBANO-MUNICIPAL PROPOSTO}

Propor um indicador de sustentabilidade que envolva o fator de carga em âmbito nacional seria inadequado porque no Brasil além de diferenças climáticas existem diferenças culturais e sociais nas regiões que refletem nos hábitos de consumo conforme demonstrado por PROCEL (2007). Tais diferenças impactariam na curva de carga dos alimentadores, tornandose mais adequada a proposição de Um ISU.

A partir disto apresenta-se na Figura 4 uma cidade fictícia que contém 3 alimentadores de média tensão, de 1 a 36,2[kV] conforme ABNT(2005), onde a partir de medidores, localizados nas pontos de análise (P-Análise-n) se torna possível coletar dados para construir as curvas de carga e efetuar o cálculo de indicadores envolvendo-as. 
Figura 4 - Cidade fictícia

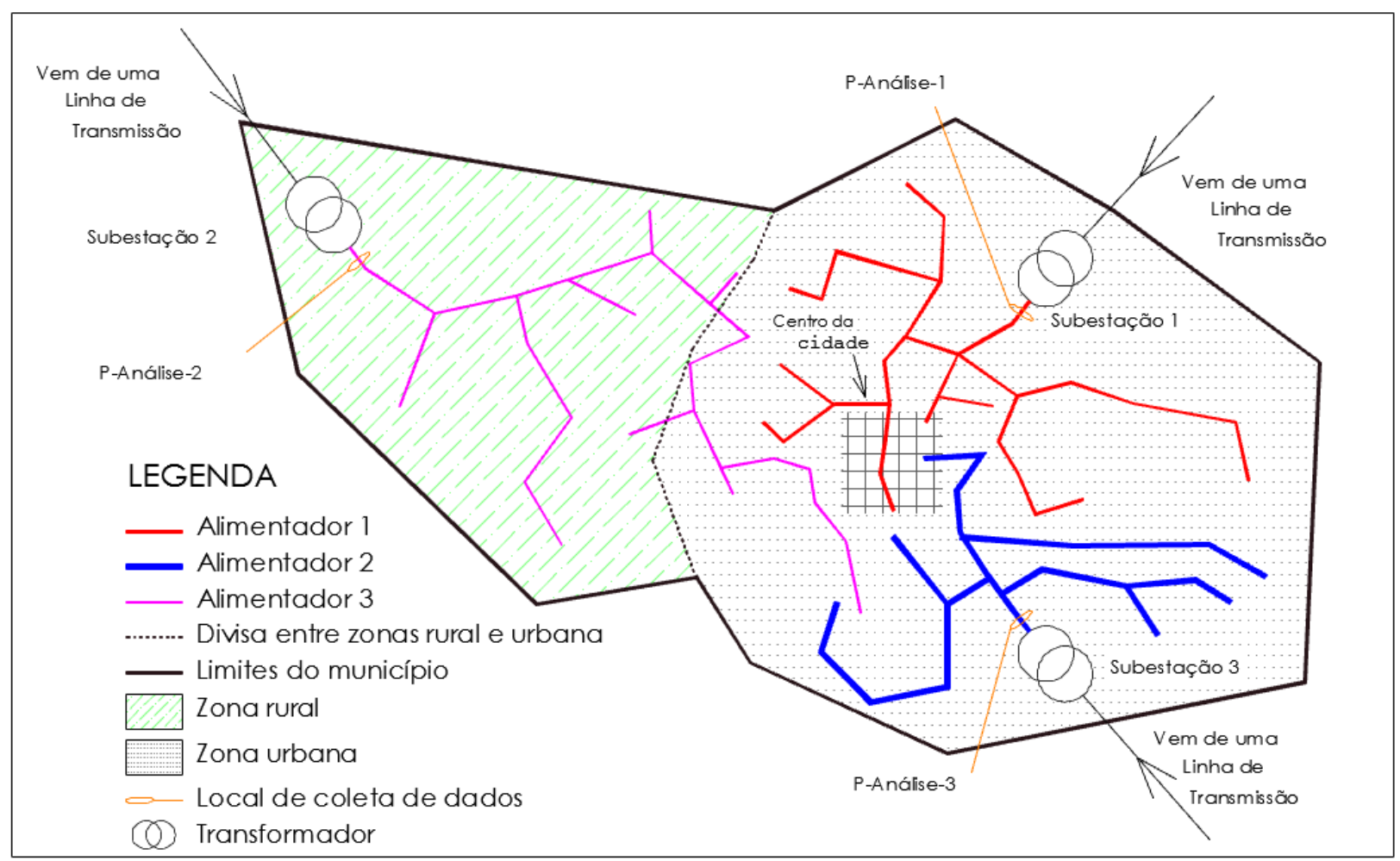

Fonte: Os autores (2019)

No modelo foram consideradas 3 subestações de energia elétrica, cada uma com uma rede elétrica de saída. Foi proposta uma ponderação do fator de carga com o intuito de aprimorar a detecção de planificação da curva de carga considerando a proporção de cada alimentador no fornecimento de eletricidade. Ademais, adicionou-se a capacidade de distribuição de energia de cada alimentador, conforme a Equação (3).

$$
f_{\text {carga-ponderado }}=\frac{\sum_{n=1}^{n}\left(f_{\text {carga-n }} \times C_{\text {sistema-n }}\right)}{\sum_{n=1}^{n} C_{\text {sistema- } n}}=\frac{\left(f_{\text {carga-ALM1 }} \times C_{\text {ALM1 }}+f_{\text {carga-ALM2 }} \times C_{\text {ALM2 }}+f_{\text {carga-ALM3 }} \times C_{\text {ALM3 }}\right)}{\left(C_{\text {ALM1 }}+C_{\text {ALM2 }}+C_{\text {ALM3 }}\right)}
$$

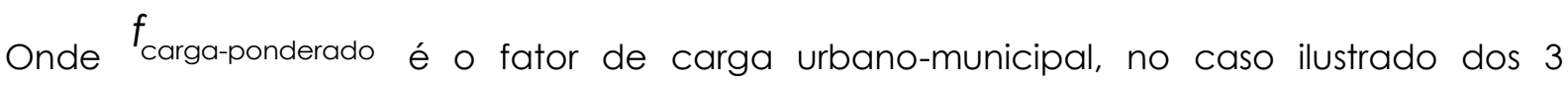

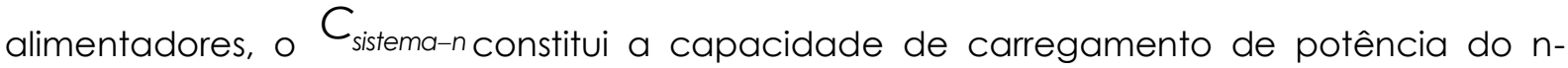
alimentador.

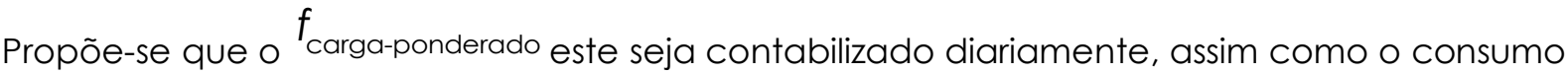
diário urbano-municipal. Dessa forma, obtém-se um Consumo Diário per capita Conjugado, considerando que a demanda na curva da Figura 3 assim como a capacidade dos alimentadores, na Equação (4) o ISU proposto é apresentado e aplicado numericamente.

$$
C_{\text {diário-per-capita-conjugado }}=\frac{C_{\text {diário-per-capita }}}{f_{\text {carga-ponderado }}}=\frac{210,45}{0,461513}=456\left[\frac{\mathrm{Wh}}{\mathrm{hab} \times \text { dia }}\right]
$$

O acréscimo da palavra conjugado sinaliza a reunião entre os aspectos do consumo per capita e a distribuição diária no uso da eletricidade. Considerando na cidade hipotética de 
curva da Figura 3, com fator de carga de 0,461513, o ISU proposto seria de 456[Wh/(hab.dia)], muito maior se a curva fosse planificada, coincidente ao valor calculado na Equação (2). Assim o formato irregular da curva de carga foi detectado pelo $C_{\text {diário-per-capita-conjugado, haja }}$ vista que quanto maior numericamente pior seria o uso de energia na cidade ou município.

Portanto o ISU proposto deve ser calculado para todos os dias do ano sendo o valor máximo ocorrido efetivado. Percebe-se que a redução do consumo de eletricidade através da eficiência energética também acarretaria a redução do proposto por consequência de seu numerador. Já a planificação da curva do município aumentaria o fator de carga ponderado e por consequência reduzindo sua amplitude.

\section{CONCLUSÕES}

A partir do exposto foi possível notar a viabilidade de uso do ISU proposto que efetue detecção global dos impactos do GLD nas cidades ou municípios desde que sejam utilizados recursos de tecnologia da informação e comunicação para obtenção e análise das curvas de carga diariamente. Tal adequação será requisito para as cidades inteligentes conforme cenário vislumbrado pelas ISO 37122 (ISSO,2019) e ISO/IEC DIS 30146 (ISO, 2019). A viabilidade do ISU proposto foi demonstrada com consistência numérica.

\section{REFERÊNCIAS}

ANEEL. Resolução $n^{\circ} 733$, de 2016 . Estabelece as condições para a aplicação da modalidade tarifária horária branca. Resolução Normativa № 733. Brasília, DF, 06 set. 2016.

ASSOCIAÇÃO BRASILEIRA DE NORMAS TÉCNICAS. NBR 14039: Instalações elétricas de média tensão de 1,0 kV a 36,2 kV. 2 ed. Rio de Janeiro: ABNT, 2005. 87 p.

ASSOCIAÇÃO BRASILEIRA DE NORMAS TÉCNICAS. NBR ISO 37120: Desenvolvimento sustentável de comunidades - Indicadores para serviços urbanos e qualidade de vida. Rio de Janeiro: ABNT, 2017. 87 p.

BELL, Simon; MORSE, Stephen. Sustainability Indicators: Measuring the Immeasurable? 2. ed. Londres: Earthscan, 2008. 228 p.

INTERNATIONAL ATOMIC ENERGY AGENCY et al. (Austria). International Atomic Energy Agency. Energy Indicators for Sustainable Development: Guidelines and Methodologies. Viena: International Atomic Energy Agency, 2005. 161 p.

INTERNATIONAL ORGANIZATION FOR STANDARDIZATION. ISO/IEC DIS 30146: Information technology — Smart city ICT indicators. 1 ed. Genebra: ISO, 2019.

INTERNATIONAL ORGANIZATION FOR STANDARDIZATION. ISO 37122:2019: SUstainable cities and communities -- Indicators for smart cities. 1 ed. Genebra: ISO, 2019. 95 p.

KAGAN, Nelson; OLIVEIRA, Carlos César Barioni de; ROBBA, Ernesto João. Introdução aos sistemas de distribuição de energia elétrica. São Paulo: Edgard Blucher, 2005. 328 p.

MUNICIPAL COUNCIL OF ENVIRONMENT AND SUSTAINABILITY (Espanha). Indicators 21: Local sustainability indicators in Barcelona (data from 2012). Barcelona: Municipal Council Of Environment And Sustainability, 2012.67 p.

PROCEL - PROGRAMA NACIONAL DE CONSERVAÇÃO DE ENERGIA ELÉTRICA (Rio de Janeiro). Eletrobrás - Centrais Elétricas Brasileiras S. A. Avaliação do Mercado de Eficiência Energética no Brasil: PESQUISA DE POSSE DE EQUIPAMENTOS E HÁBITOS DE USO - ANO BASE 2005 -CLASSE 
RESIDENCIAL RELATÓRIO BRA. Rio de Janeiro: Eletrobras / Procel, 2007. 185 p. Disponível em: <www.procelinfo.com.br/>. Acesso em: 07 set. 2017.

REDE NOSSA SÃO PAULO (SP). Rede Nossa São Paulo. Programa Cidades Sustentáveis. 5. ed. São Paulo: Gráfica Interfill, 2012. Disponível em:

<http://www.cidadessustentaveis.org.br/downloads/publicacoes/publicacao-programacidades sustentaveis.pdf>. Acesso em: 20 maio 2018.

SILVA, Sandra R. M.; SHIMBO, Ioshiaqui. INDICADORES DE SUSTENTABILIDADE URBANA. In: ENTAC 2000 - ENCONTRO NACIONAL DE TECNOLOGIA NO AMBIENTE CONSTRUÍDO, 8., 2000, Salvador. Anais do VIII ENTAC. Salvador: Anac, 2000. p. 1 - 8. Disponível em:

<http://www.infohab.org.br/entac2014/2000/Artigos/ENTAC2000_606.pdf>. Acesso em: 27 maio 2018.

SMART ENERGY DEMAND COALITION (Belgica). SEDC. Mapping Demand Response in Europe Today: 2015. Bruxelas: SEDC, 2015. Disponível em: <www.smartenergydemand.eu>. Acesso em: 20 abr. 2018.

SMART ENERGY DEMAND COALITION (Belgica). SEDC. Mapping Demand Response in Europe Today: 2017. Bruxelas: SEDC, 2017. Disponível em: <www.smartenergydemand.eu>. Acesso em: 20 abr. 2018.

SUSTAINABLE SEATTLE (Washington Usa). Sustainable Seattle. Indicators of Sustainable Community: A status report on long-term cultural, economic, and environmental health for Seattle/King County. Seattle: Sustainable Seattle, 1998. 74 p.

WONG, Steven. Canadian Residential Demand Response and Ancillary Service Market Opportunities. Varennes: Canmetenergy - Varennes Research Center, 2015. 57 p. 